\title{
Opening windows for bone remodeling through a SLIT
}

\author{
Jameel Iqbal, ${ }^{1,2}$ Tony Yuen, ${ }^{1}$ Se-Min Kim, ${ }^{1}$ and Mone Zaidi ${ }^{1}$ \\ 'Mount Sinai Bone Program, Icahn School of Medicine at Mount Sinai (ISMMS), New York, New York, USA. Department of Pathology, James J. Peters VA Medical Center, New York, New York, USA.
}

\begin{abstract}
Bone formation and resorption are tightly coupled, and dysfunction of either process leads to bone diseases, such as osteoporosis. Bone-forming agents have been explored clinically to increase bone density; however, long-term efficacy of these strategies is limited due to the accompanying increase in resorption in response to increased bone formation. Axonal guidance molecules have recently been shown to regulate formation-resorption coupling and thus have the potential for osteoporosis therapy. In this issue of the JCI, Kim et al. demonstrate that osteoclast-secreted SLIT3 influences bone formation and resorption by promoting osteoblast migration and suppressing osteoclast differentiation. Activation of SLIT3/ROBO signaling in ovariectomized mice increased bone mass, suggesting that SLIT3 should be further explored as a therapeutic target.
\end{abstract}

\section{A fine balance: bone formation and resorption}

Bone is a dynamic vital organ that constantly responds to external stimulation and stress; however, in the absence of mechanical stimulation, such as astronauts experience in the zero gravity of space, bone breaks down (1). Likewise, the mechanisms that keep bones strong and healthy ultimately succumb in old age, resulting in osteoporosis, a leading cause of morbidity and fractures in the elderly (2). In 1786, John Hunter, an orthopedic surgeon, suggested that osteoporosis results from a defect in bone remodeling, a process wherein old bone is constantly replaced by equal quanta of new bone such that there is no net bone loss under normal conditions. It was later shown that bone remodeling is an asynchronous process, whereby bone resorption, mediated by multinucleated osteoclasts, is followed by bone formation via groups of osteoblasts (3). However, only recently has this temporal and spatial asynchrony been visualized in vivo (4).
Strategies to target certain molecules that control the differentiation and function of osteoblasts and osteoclasts have been implemented for osteoporosis therapy. Among the most successful approaches has been the use of denosumab, a mAb that blocks the essential action of the TNF superfamily cytokine RANKL on osteoclast formation, which is now a standard therapy for osteoporosis (5). However, denosumab and other approved osteoporosis therapies suffer from one critical drawback: their action is limited by tight coupling of formation and resorption. Almost every stimulus that reduces bone resorption also invariably reduces bone formation, and vice versa (6). Thus, when bone-forming agents, such as parathyroid hormone (teriparatide) or parathyroid hormone-related protein (abaloparatide) are used therapeutically, bone formation increases, but bone resorption also rises, thus limiting the anabolic action of these agents to a window of efficacy of about

Related Article: p. 1429

Conflict of interest: MZ has served as an expert witness for Roche Pharmaceuticals. MZ is an inventor on an issued US patent (US8435948B2) relating to the effects of FSH inhibitors on bone and is an inventor on pending patent applications submitted by the ISMMS on the effects of inhibiting FSH action on body fat. In the case of commercialization, MZ will be the recipient of royalties and other income per ISMMS policies.

Reference information: J Clin Invest. 2018;128(4):1255-1257. https://doi.org/10.1172/JCI120325.

two years. Likewise, antiresorptive agents, such as bisphosphonates and denosumab, suppress bone resorption, but also reduce bone formation - a clinical concern that often limits their long-term use.

The cataloging of human mutations and the use of genetically modified mice have together unraveled molecules, such as TGF- $\beta$ and IGF-1, that control resorption-formation coupling (7). Many diffusible axon guidance molecules, such as neurotrophins, semaphorins (SEMAs), and netrins, which normally function as chemoattractants or chemorepellents for growing nerve fibers, are expressed in osteoblasts and osteoclasts (8). It is now clear that these neural proteins are repurposed by the skeleton to control the coupling of bone resorption to bone formation (9) (Figure 1).

\section{Role for SLIT3 in coupling bone formation and resorption}

In this issue, Kim et al. show that the axonal guidance molecule SLIT3 is secreted by osteoclasts to regulate resorption-formation coupling (10). Vertebrates express three SLIT proteins (SLIT1, SLIT2, and SLIT3), each of which is composed of four leucine-rich repeat (LRR) domains, numerous EGF repeats, an agrin-perlecan-laminin-slit (APLS, also known as laminin-G-like) domain, and a C-terminal cysteine knot (11). SLITs can homodimerize through their LRR4 domain and bind roundabout (ROBO) receptors via the LRR2 domain. ROBO receptors were named for the commissural axon roundabout observed at the brain midline following genetic deletion of Robo (11).

In the nervous system, SLITs serve as chemorepellents and act in coordination with molecules, such as SEMAs and netrins, to guide and stabilize axons. These same molecules have been discovered as serving as cocoordinators of other processes, such as angiogenesis, and importantly, bone formation and resorption coupling. SEMA3a has been shown to inhibit activation of RhoA and osteoclastogenesis, while at the same time, to induce Rac activation to enhance $\beta$-cat- 


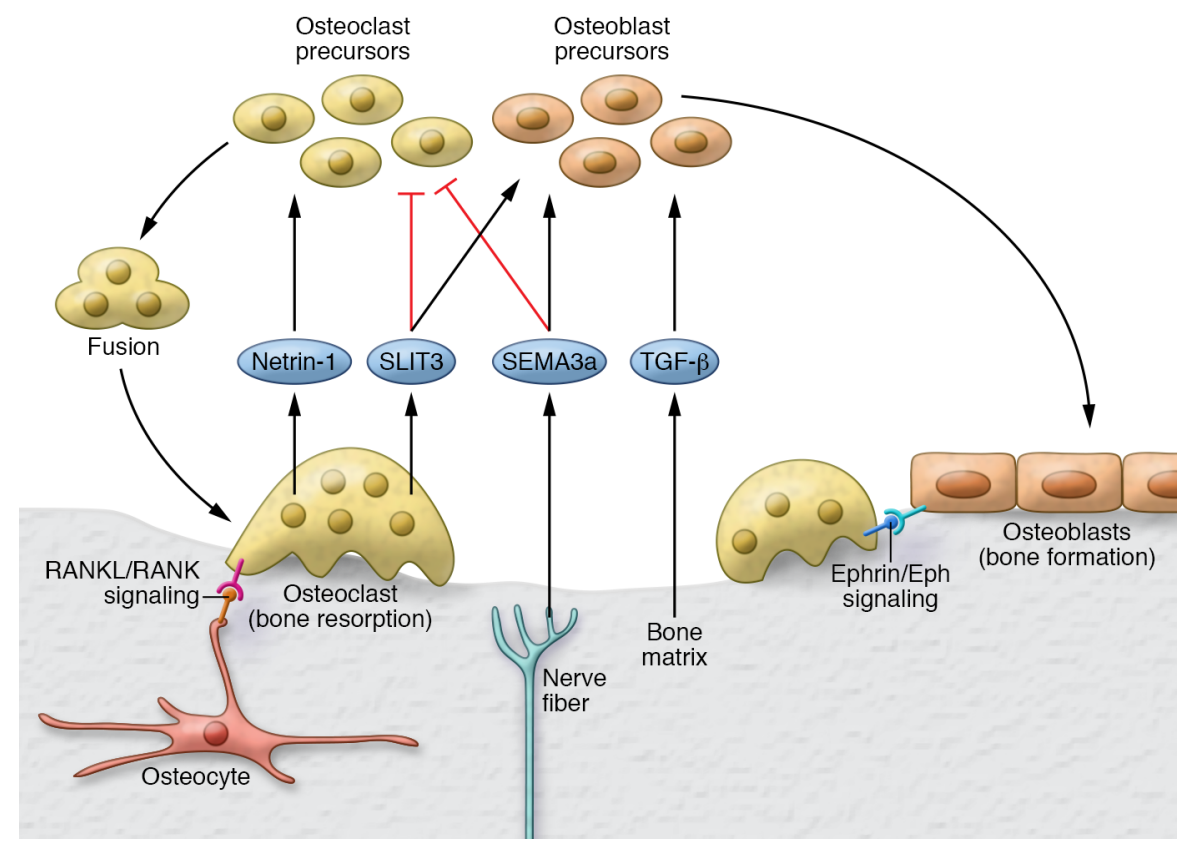

Figure 1. Role of axonal guidance molecules in bone formation and resorption coupling. Bone formation and resorption coupling is a process wherein osteoclastic bone resorption is followed by osteoblastic bone formation. For resorption, two classes of axon guidance molecules, SLITS (SLIT3) and semaphorins (SEMA3a), act together to fine-tune repulsive signals for osteoclast migration, while inhibiting differentiation and cell fusion. SLIT3 is produced by mature osteoclasts, whereas the critical bone actions of SEMA3a originate from nerves. These repulsive and inhibitory effects of SEMA3a and SLIT3 on osteoclasts are countered by netrin-1, another molecule originally identified for its role in axon guidance. Netrin-1 attracts osteoclasts and promotes their fusion. Additional molecular pathways controlling osteoclasts include ephrin/ephrin receptor tyrosine kinase (ephrin/EPH) signaling via cell contact between osteoblasts and osteoclasts, and the RANKL/RANK pathway for osteoclastogenesis. For bone formation, signals released from mineralized matrix, such as TCF- $\beta$ and IGF-1, in combination with signals secreted by osteoclasts, such as SLIT3, and those released by nerves, namely SEMA3a, serve in concert to recruit osteoblasts and promote their differentiation.

enin-mediated osteoblast differentiation (12). Netrin-1 opposes SEMA3a action in bone by interacting with the Unc5b receptor to enhance osteoclastic RhoA activation and early cell fusion, ultimately promoting the formation of mature giant multinucleated osteoclasts (9).

Kim et al. found that, similarly to SEMA3a, SLIT3 acts to suppress osteoclast differentiation. They demonstrated that osteoclast-derived SLIT3 acts in an autocrine and paracrine manner via binding to ROBO1 and ROBO3 to inhibit Rac activation and dendritic cell-specific transmembrane protein-mediated (DCSTAMP-mediated) early osteoclast cell fusion (10). Of note, however, is that there are several key mechanistic differences between SLIT3 and SEMA3a action on bone. For example, while SEMA3a preferentially inhibits the activation of RhoA, but not of Rac (12), Kim et al. determined that SLIT3 suppresses Rac without affecting RhoA in osteoclasts (10). Another key difference is that abnormal sensory neural input appears to underlie SEMA3a action on bone, as only neural cell-specific Sema3a deletion, and not osteoblast- or osteoclast-specific deletion, results in a bone phenotype (13). In contrast, only osteoclast-specific deletion of Slit3, and not neural cell- or osteoblast-specific deletion, produced a bone phenotype (10). Thus, the two classes of molecules act in a nonredundant, but concerted manner to fine-tune the inhibitory signals for early osteoclast migration, differentiation, and cell fusion. These repulsive effects of SEMA3a and SLIT3 are likely countered in vivo by the stimulatory effects of netrin-1, which augments osteoclast migration and promotes cell fusion (9). In osteoblasts, SLIT3 enhanced cell migration and $\beta$-catenin-mediated differ- entiation (10), a result that contrasts with a prior study of SLIT2, wherein SLIT2/ ROBO signaling was found to inhibit osteoblast differentiation (14). Further investigation is needed to decode the relative roles of the different SLIT proteins and their ROBO receptors in osteoblasts, as it is likely that the effects of SLIT3 are similar to those of SEMA3a in inducing osteoblast differentiation by augmenting $\beta$-catenin activation (12).

These new-found functions of axonal guidance molecules as controllers of bone formation and resorption coupling prompt the search for drugs with the potential to elevate bone formation and simultaneously reduce bone resorption. As proof-of-concept, Kim et al. injected a truncated SLIT3 (LRRD2) containing the ROBO-binding LRR2 domain into ovariectomized mice. LRRD2 administration reversed ovariectomy-induced bone loss and, in histomorphometric studies, increased bone formation rates and decreased eroded bone surfaces and the number of multinucleated osteoclasts (10). Likewise, SEMA3a injection reversed ovariectomy-induced bone loss by augmenting bone formation and reducing resorption (6). Together, SLIT3 and SEMA3a represent a new class of putative antiosteoporotic agents that can decouple resorption from formation, a clear therapeutic advantage.

\section{Conclusions and future directions}

Thus far, research has focused on discovering and defining how a single molecule, be it SEMA3a, netrin-1, or SLIT3, works to decouple bone formation and resorption. Future investigations should focus on how these mediators coordinate formation and resorption coupling in concert. For example, in neurons, the upregulation of SLIT causes loss of the netrin attractant response via ROBO (15). It remains unclear, however, whether similar ligand-ligand crosstalk occurs in bone cells and, if so, whether there is regulatory complexity that determines final outcome. Furthermore, in axons, ROBO receptors are modulated at the protein level (15), whereas Kim et al. primarily relied on mRNA levels as a measure of ROBO expression. It is likely that any intricate interplay between guidance molecules and their receptors will be teased out carefully 
at the cell-surface level. Similarly, while not investigated by Kim et al., the cleaved C-terminal portion of SLIT, designated SLIT-C, can bind to plexin-A1 (11), which is part of the receptor complex that mediates the action of SEMA3a on osteoclasts. It remains to be determined exactly how SEMA3a and SLIT3 work together to coordinate cell repulsion and inhibition.

Future studies should also focus on how SEMA3a, SLIT3, and/or netrin-1 interact with cell-contact and ECM molecules and whether such contact can modulate the overall responses. Recent studies show that osteoclasts in contact with osteoblasts are nonresorptive and become motile to seek out an area where they can resorb bone (4). How do secreted guidance molecules and known cell-contact coupling factors, such as ephrins, interact to direct migration and differentiation? Similarly, SLITs can bind to many ECM molecules linked to genetic bone disorders. Among these SLIT-interacting molecules are dystroglycan, which is implicated in limb-girdle muscular dystrophy, and glypicans, which are associated with osteoporotic risk in GWAS (16-18). Exploring how coupling factors interact with ECM molecules will likely reveal added avenues for treatment of ECM-related bone diseases in general and of osteoporosis in particular.

\section{Acknowledgments}

Work at ISMMS was/is supported by $\mathrm{NIH}$ grants R01 DK113627, DK80459; and R01 AG40132, R01 AG23176, R01 AR65932, and R01 AR67066 (to MZ).

Address correspondence to: Mone Zaidi, Endocrinology, PO 1055, Icahn School of Medicine at Mount Sinai, One Gustave L. Levy Place, New York, New York 10029, USA. Phone: 212.241.8797; Email: mone. zaidi@mssm.edu.

1. Iqbal J, Zaidi M. Molecular regulation of mechanotransduction. Biochem Biophys Res Commun. 2005;328(3):751-755.

2. Iqbal J, Sun L, Zaidi M. Coupling bone degradation to formation. Nat Med. 2009;15(7):729-731.

3. Hattner R, Epker BN, Frost HM. Suggested sequential mode of control of changes in cell behaviour in adult bone remodelling. Nature. 1965;206(983):489-490.

4. Furuya M, et al. Direct cell-cell contact between mature osteoblasts and osteoclasts dynamically controls their functions in vivo. Nat Commun. 2018;9(1):300.

5. Iqbal J, Sun L, Zaidi M. Denosumab for the treatment of osteoporosis. Curr Osteoporos Rep. 2010;8(4):163-167.

6. Zaidi M, Iqbal J. Translational medicine: Double protection for weakened bones. Nature. 2012;485(7396):47-48.

7. Tang Y, et al. TGF- $\beta 1$-induced migration of bone mesenchymal stem cells couples bone resorption with formation. Nat Med. 2009;15(7):757-765.
8. Togari A, Mogi M, Arai M, Yamamoto S, Koshihara Y. Expression of mRNA for axon guidance molecules, such as semaphorin-III, netrins and neurotrophins, in human osteoblasts and osteoclasts. Brain Res. 2000;878(1-2):204-209.

9. Mediero A, Ramkhelawon B, Perez-Aso $\mathrm{M}$, Moore KJ, Cronstein BN. Netrin-1 is a critical autocrine/paracrine factor for osteoclast differentiation. J Bone Miner Res. 2015;30(5):837-854.

10. Kim BJ, et al. Osteoclast-secreted SLIT3 coordinates bone resorption and formation. J Clin Invest. 2018;128(4):1429-1441.

11. Blockus H, Chedotal A. Slit-Robo signaling. Development. 2016;143(17):3037-3044.

12. Xu R. Semaphorin 3A: A new player in bone remodeling. Cell Adh Migr. 2014;8(1):5-10.

13. Fukuda $\mathrm{T}$, et al. Sema3A regulates bone-mass accrual through sensory innervations. Nature. 2013;497(7450):490-493.

14. Sun H, Dai K, Tang T, Zhang X. Regulation of osteoblast differentiation by slit 2 in osteoblastic cells. Cells Tissues Organs. 2009;190(2):69-80.

15. Stein E, Tessier-Lavigne M. Hierarchical organization of guidance receptors: silencing of netrin attraction by slit through a Robo/DCC receptor complex. Science. 2001;291(5510):1928-1938.

16. Wright KM, Lyon KA, Leung H, Leahy DJ, Ma L, Ginty DD. Dystroglycan organizes axon guidance cue localization and axonal pathfinding. Neuron. 2012;76(5):931-944.

17. Liang Y, et al. Mammalian homologues of the Drosophila slit protein are ligands of the heparan sulfate proteoglycan glypican-1 in brain. J Biol Chem. 1999;274(25):17885-17892.

18. Kemp JP, et al. Identification of 153 new loci associated with heel bone mineral density and functional involvement of GPC6 in osteoporosis. Nat Genet. 2017;49(10):1468-1475. 